\title{
AN INVERSE FREE ELECTRON LASER ACCELERATOR EXPERMMENT
}

Iddo Wemick and T.C. Marshall

Department of Applied Physics, Columbia University, New York, NY 10027

\section{ABSTRACT}

A free electron laser was configured as an autoaccelerator to test the principle of accelerating electrons by stimulated absorption of radiation $(\lambda=1.65 \mathrm{~mm})$ by an electron team $(750 \mathrm{kV})$ traversing an undulator. Radiation is produced in the first section of a constant feriod undulator $\left(l_{W 1}=1.43 \mathrm{~cm}\right)$ and then absorbed $(-4 C \%)$ in a second undulator, having a tapered period $\left(l_{\mathrm{w} 2}=1.8-2.25 \mathrm{~cm}\right)$, which results in the acceleration of as sutgoup $(-0.9)$ of elections to $-1 \mathrm{MeV}$.

The principle of using free electron laser [FEL] physics to accelerate electrons 1.. Is Strited by Palmer ${ }^{1}$ in 1972, however despite the extensive development of the E. $\quad \therefore$ ere has been no demonstration of the stimulated absorption of a laser pulse a camanied by acceleracion of a group of electrons while the electron beam is aresio an unisulator [FEL]. The idea has been re-examined in more detail ${ }^{2-4}$ and

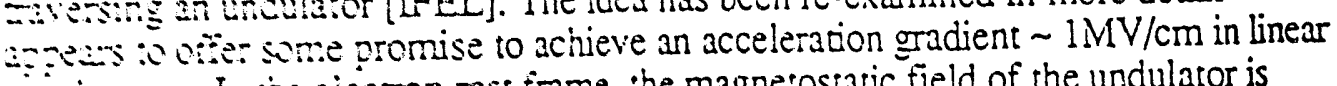

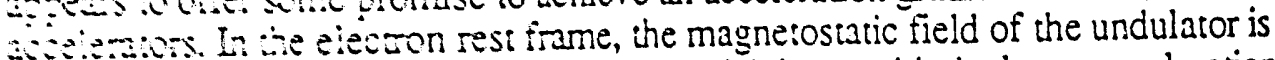

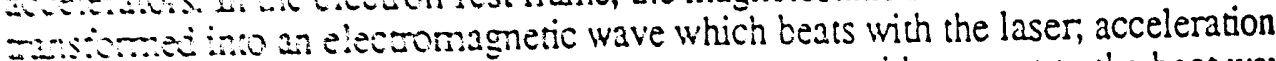
a:us sy jeaz: sthe phase of the electons constant with respect to the beat wave, by

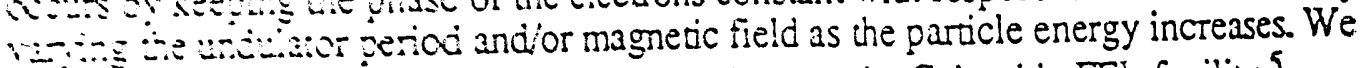
tar Eaves a relsively simple experiment, done on the Columbia FEL faciliry ${ }^{5}$, which iemons azes thai acce!erajon does occur.

Tre Coimmia FEL operates at a wavelength of $\sim 1.6 \mathrm{~mm}$ and produces about Five IN tom $3.50 \mathrm{kV}$ election beam. As there is no powerful laser source at this wavelength, we have configured the experiment as an "autoaccelerator" [IFELA] in which a subouro of electrons is accelerated by the "inverse FEL" mechanism at the expense of the average energy of the entire bearn. This is done by separating the undulator inio two sections.

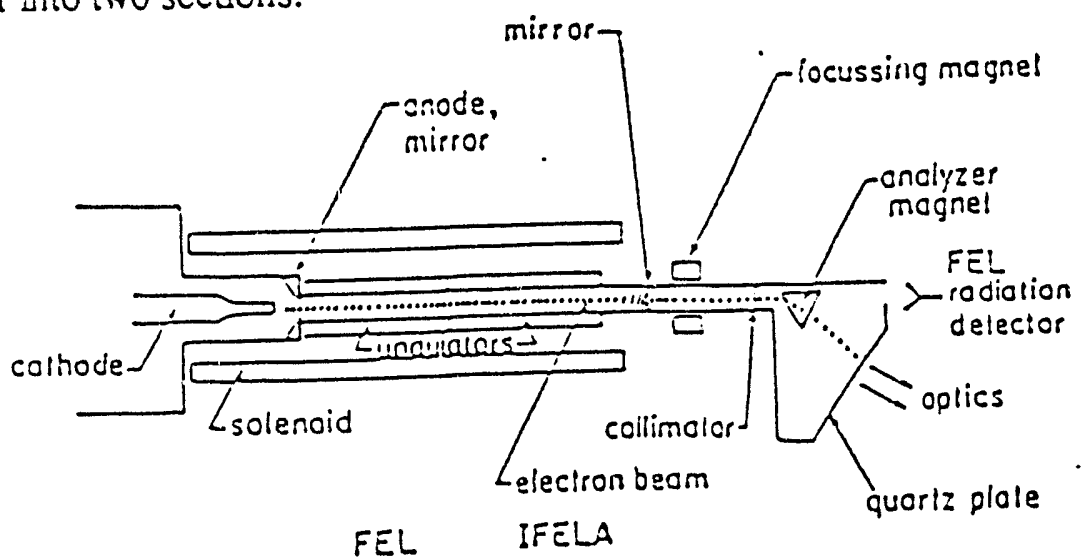

Figure 1: Schematic of the IFELA and the magnetic spectrometer. Electron emission $\propto c c u r s$ from a cold graphite cathode in a field-immersed diode, the beam is formed by a $4 \mathrm{~mm}$ dia. aperture in a graphite anode, which also serves as the upstream mirror of the resonator.

$$
\begin{aligned}
& \text { Theid Wulish op it aderaced A colet abou Concepts } \\
& \text { Pat Oeffecion, } v \text { Y 14-de Gume } 1952
\end{aligned}
$$


The first section [Fig 1] together with a pair of $75 \%$ reflecting coaxial mirrors develops FEL radiation which grows from noise to saturated intensity and causes a bunching of the electrons. These particies then enter the second section of the undulator where the period is increased and then tapered along the axis so that a subgroup of electrons is accelerated as stimulated absorption of the wave occurs. We report measurements of this nonlinear absorption together with the electron energy spectrum. The accelerator section acts as a luad for the oscillator, but its absorption is not high enough to prevent oscillation of the entire system.

Table I summarizes the parameters of the IFELA experiment. The undulator is a bifilar helical winding which provides a transverse field of order $600 \mathrm{G}$ following an adiabatic entry region.

Table I : Operating Conditions of the Columbia IFELA

Beam Energy

Beam Diameter

Beam current

Pulse length

Drift tube diameter

Undulator: first section

Undulator: second section

\author{
$750-800 \mathrm{kV}$ \\ $4 \mathrm{~mm}$ \\ $150 \mathrm{~A}$ \\ $150 \mathrm{nsec}$ \\ $11 \mathrm{~mm}$ \\ $l_{W} 1=1.4 \mathrm{~cm}$ \\ $\mathrm{B \perp}=600 \mathrm{G}$ \\ length, $40 \mathrm{~cm}$ \\ $l_{W 2}=1.8-2.25 \mathrm{~cm}$ \\ taper $=\frac{1}{I_{W}} \frac{d_{l_{W}}}{d z}=0.0067 \mathrm{~cm}^{-1}$ \\ $\mathrm{B} \perp=400 \mathrm{G}$ \\ length $=37.5 \mathrm{~cm}$
}

The beam is guided and focused along the drift tube by a uniforn solenoidal field $\sim \mathrm{TT}$ which causes "Grolv" !" orbits 6 . The FEL power examined by a grating spectrometer shows a carrier wavelength of $1.6 \mathrm{~mm}$ together with a pair of sidebands which carry about one-third of the total power, it was found that only the carrier was absorbed by the acceleration process ${ }^{7}$. The downstream mirror is polished graphite with a small hole on its axis followed by a collimator which forms the objective of the electron beam optics. A focusing solenoid guides the electrons beyond the fringe field of the solenoid. A dipole field using triangular polefaces deflects the beam and disperses the electrons onto a quartz viewing plate where the impact causes substantial Cerenkov light 8 (the quartz is painted with an opaque graphite film on the vacuum side). The light from the energetic electrons is directed to two lead-shielded photomultipliers located in a shielded room with the other electronics. The magnetic spectrometer is calibrated by using the electron beam with no undulator. The use of two photocells permits one photocell to monitor the principal group near the injection energy while the other scans the energy channels for the accelerated electrons.

The undulator in the IFELA section is designed using a numerical model to choose the appropriate field and taper. We use a set of equations 5 that models the electron motion in $1 \mathrm{D}$ along a single pass through the system and a self-consistent set of $2 \mathrm{D}$ field equations. 


$$
\begin{aligned}
& \frac{d \psi_{i}}{d z}=k_{w}+k_{s}-\frac{k_{s}}{\sqrt{1-\frac{1+a_{w}^{2}+2 a_{w} a_{s} \cos \psi_{i}}{\gamma_{i}^{2}}}+\frac{\partial \Phi}{\partial z}} \\
& \frac{d \gamma_{i}}{d z}=-\frac{a_{w} a_{s} k_{s}}{\gamma_{i}} \sin \psi_{i}+\frac{2 \omega_{p}^{2}}{k_{s} c^{2}}\left[<\cos \psi>\sin \psi_{i}-<\sin \psi p \cos \psi_{i}\right] \\
& \left(\nabla_{\perp}{ }^{2}+2 i k_{s} \frac{\partial}{\partial z}+2 i \frac{\omega}{c^{2}} \frac{\partial}{\partial t}\right) u(r, t)=-F \frac{\omega_{p} a_{w}}{c^{2}}<\frac{e^{-(\psi-\Phi)}}{\gamma}>
\end{aligned}
$$

Equation 1 is used to determine the phase $\Psi$ of the electrons with respect to the signal wavefront as the electrons traverse the undulator. Equation 2 is the energy equation for the electrons and finally equation 3 is the $2 \mathrm{D}$ wave equation which describes the signal growth. The "Raman" term (i.e. terms $\propto \omega_{p}^{2}$ ) which accounts for the longitudinal space charge electric field from the electron bunches is included and is necessary to obtain the correct growth rate in the FEL section and the correct interaction in the IFELA section. The electron beam in the experiment has normalized parallel momentum spread $\sim 1 \% 9$, and is represented in the numerical study as a "cold" beam, since the trapping width, $\Delta \gamma / \gamma \propto \sqrt{a_{s} a_{w}}$ where as and aw are the normalized vector potentials of the signal and undulator fields, is much larger than $1 \%$.

The magnetic field of the FEL undulator section was chosen so that the power would grow roughly a factor of 25 in $40 \mathrm{~cm}$. This gain will sustain oscillation and results in a signal which reaches power saturation at the point where the electrons enter the IFELA section. The saturated power intensity on the beam axis, $10 \mathrm{MW} / \mathrm{cm}^{2}$, is consistent with the power output from an FEL oscillator device very similar to this one ${ }^{10}$. It is found numerically that the wave amplitude is reduced by one-half as it reaches the end of the IFELA undulator as a subgroup of the trapped electrons is accelerated to $1 \mathrm{MV}$. The code includes no slippage and therefore does not account for sideband radiation; hence the measured absorption of the FEL power by the accelerator module will be less than the model predicts. The taper of the undulator which optimizes the absorption and acceleration is found through trial and error of the numerical study and corresponds to an acceleration gradient of $\sim 7 \mathrm{kV} / \mathrm{cm}$ in this test experiment. The taper is used to generate a variable-period helix which is mapped onto a section of phenolic tube and then cut to specification "by hand". Measurements of the actual undulator period taper and magnetic field taper are then incorporated into the code to simulate the actual experimental situation. Once the taper is chosen, a series of simulations show that the acceleration is not very sensitive to variation in FEL power or undulator field, however, increasing the power input to the IFELA section will accelerate more electrons. The field in the IFELA undulator can be varied independently of the FEL undulator, both are powered by a capacitor bank discharge synchronized to the accelerator timing system.

A set of representative data is shown in Fig 2. Shots are selected for a relatively flat diode voltage history, with electron energy near resonance. A determining signature as the electron beam energy reaches the design value is a 
decrease of transmitted FEL power accompanied by an increase of light signal in the photomultipliers which respond to the accelerated electrons. The energy bins are separated by the resolution of the electron bearn optics. Background light, obtained from operation of the apparatus with zero undulator field, is subtracted from the signal. The transmitted FEL power is monitored by a Schottky-barrier diode detector located

(a)

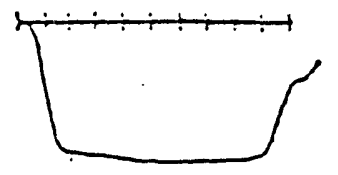

(b)

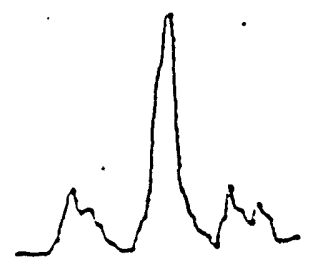

(c)

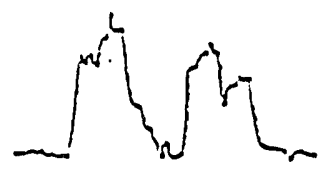

Figure 2. Representative signals obtained in the experiment. (a) Accelerator voltage, $-800 \mathrm{kV}$ maximum; (b) signal from photocell monitoring $1 \mathrm{MV}$ electrons, showing a burst of electrons at middle of trace; (c) FEL power transmitted through the IFEL stage, showing absorption in middle of trace; 20nsec/div, horizontal scale.

along the beam axis. It is found that the fraction of power absorbed does not depend sensitively upon the power level itself. This behavior is to be expected from the model, which shows that increases in power absorbed result in more accelerated electrons but not necessarily higher electron energy.

Figure $3 a$ displays results from a numerical simulation of the experiment showing the growth of the FEL signal, followed by the attenuation of the wave in the accelerator section. Fig 36 shows experimental data giving the FEL power transmitted through the accelerator section. This shows that there is a reduction of emitted power by $\sim 40 \%$ in the vicinity of the resonant energy of the design. By reducing the undulator field in the accelerator section to $250 \mathrm{G}$, the amount of observed abscrption decreases to about $25 \%$ of the incident power. The study of the FEL spectrum 7 shows that the sidebands of the FEL power spectrum are not absorbed by the accelerator to a measurable amount, and therefore the reduction of the incident carrier intensity $[-75 \%]$ indicated in the numerical simulation is larger than would be obtained if the sideband power is included in the measurement, as was the case.

Figure 4 shows the measured electron energy spectrum. The data is compared with the numerical simulation, run according to the experimental conditions. The cornputed spectrum is processed so that the ordinate corresponds to the number of simulation electrons contained in a bin having the same width as the experimental bin. The relative number of electrons accelerated and the acceleration energy are in good agreement with the numerical model. According to numerical simulation the smaller peak which occurs at $\gamma=2.2$ does not result from the acceleration process. 

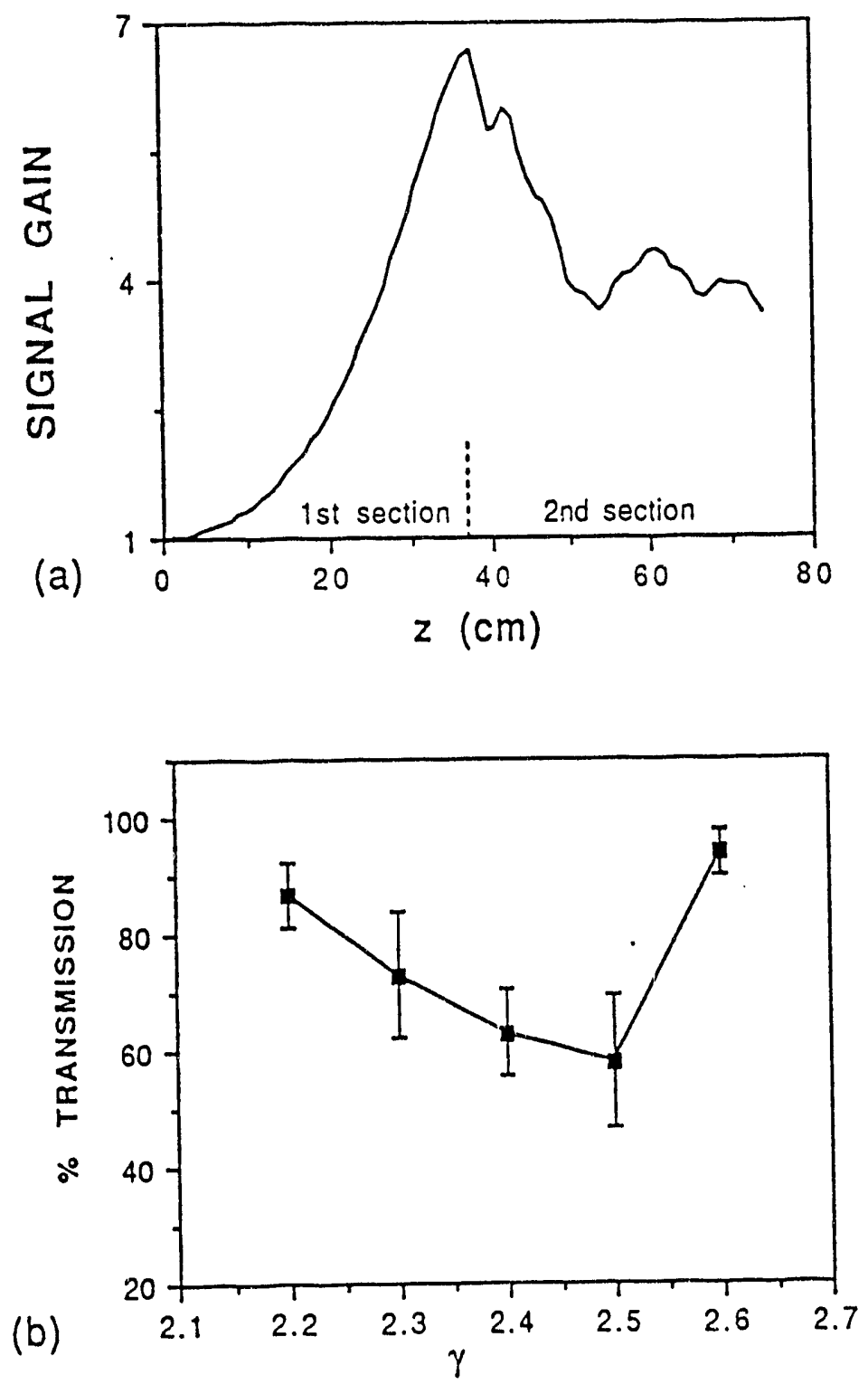

Figure 3. (a) Numerical result showing growth of FEL signal (initial intensity $=0.3 \mathrm{MW} / \mathrm{cm} 2$ ) and attenuation in the IFELA section (begins at $z=37.5 \mathrm{~cm}$ ); (b) Radiation transmitted through the IFELA section as a function of initial electron beam energy. Parameters of simulation taken from Table I. Error bars indicate standard deviation of the data. 


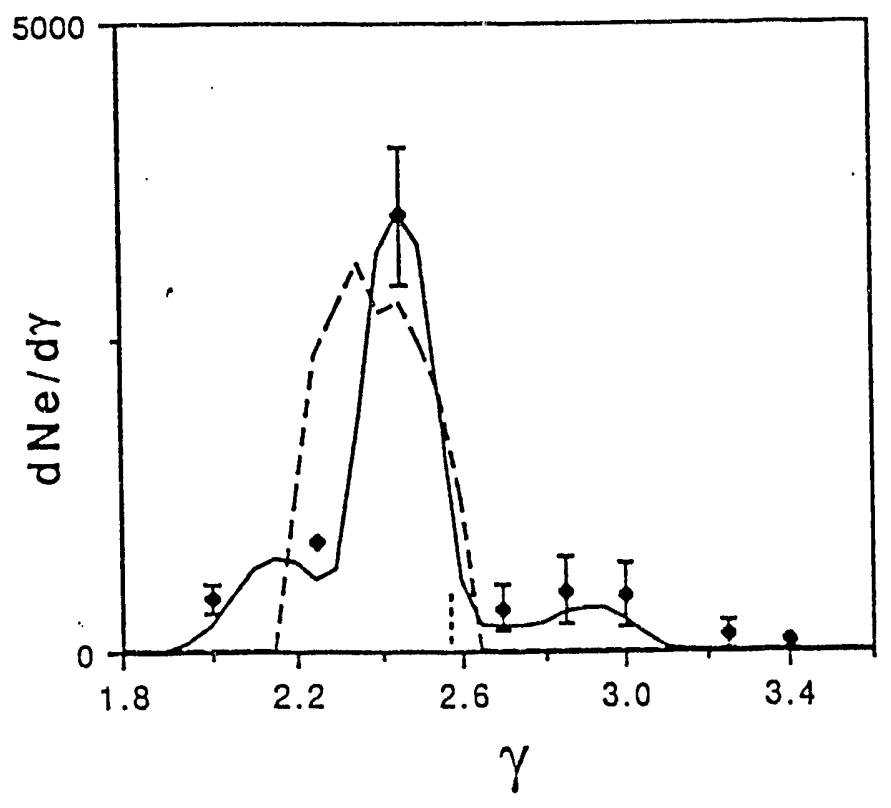

Figure 4. Measured electron energy spectrum(solid points);Electron energy spectrum obtained from simulation, at the exit from the IFELA(solid line); Electron energy spectrum obtained from simulation, at entry to the IFELA section(dashed line). The dotted vertical line denotes the injection energy.

For comparison, the dashed line on Fig 4 shows the electron spectrum computed (but not observed) at the end of the FEL section only; one notes there is a rapid cutoff of electrons having energy in excess of $\gamma=2.7$, which is well below the energy of the accelerated group. Thus electrons at $\sim 1 \mathrm{MV}$ have energy beyond the broadened distribution which results from the bunching and trapping of electrons from the FEL interaction. Electrons above the injection energy in FELs have been observed 11,12 however they are not "accelerated" by the IFEL mechanism. The number of accelerated electrons is about $9 \%$ of the total number, as can be estimated from Fig 4 . The ratio of the power required to accelerate these electrons to the overall power of the electron beam is about $3 \%$, which is less than the efficiency of the FEL (4-6\%).

In an actual IFEL where the radiation is supplied by an extemal laser, the undulator field can be considerably larger (roughly a factor of ten greater than in our experiment) and the intensity of the laser wave can be higher by perhaps a factor of a thousand. Taking a $10.6 \mu$ laser wavelength as an ex.ample our observed acceleration gradient $\sim 7 \mathrm{kV} / \mathrm{cm}$ would scale up by a factor of $\sim 100$ for such a device. Techniques exist to control synchrotron radiation losses which are no longer negligible at high energy 3 . Our success with the experiment and its interpretation suggests that the IFEL is a promising technology for an "advanced accelerator" demonstration.

\section{ACKNOWLEDGMENT}

This research is supported by the NSF, grant ECS -89-13066, DOE grant 0291ER40669, and ONR grant N00014-89-J-1652. The assistance of $Y-P$ Chou in the early stages of this project is greatly appreciated. 


\section{REFERENCES}

i. R. B. Palmer, J. Appl. Phys. 43, 3014 (1972).

2. P. Sprangle and C. M. Tang, IEEE Nucl. Sci. NS-28, 3346 (1981).

3. E. D. Courant, C. Pellegrini, and W. Zakowicz, Phys. Rev. A32, 2813 (1985).

4. A. van Steenbergen and C. Pellegrini, private commun.

5. A. Bhattacharjee, S. Y. Cai, S. P. Chang, J. W. Dodd, A. Fruchtman, and T. C. Marshall, Phys. Rev. A40, 5081 (1989).

6. L. Friedland, Phys.' Fluids 23, p.2376 (1980).

7. T. C. Marshall, A. Bhattacharjee, S. Y. Cai, Y. P. Chou, and I. Wernick, Nuclear Instruments and Methods in Physics Research A304, 683 (1991).

8. B. Kulke, M. J. Burns, and T. J. Orzechowski, LLNL Report UCRL-95598.

9. S. C. Chen and T. C. Marshall, IEEE J. Quantum Electronics QE-21, 924 (1985).

10. F. G. Yee, T. C. Marshall, and S. P. Schlesinger, IEEE Trans. on Plasma Science 16, 162 (1988).

11. A. H. Lumpkin and R. B. Feldman, Nucl. Instruments and Methods in Phyzics Research, A2.59, 19 (1987).

12. T. I. Smith, J. C. Frisch, R. Rohatgi, H. A. Schwettman, and R. L. Swent, Nucl. Instruments and Methods in Physics Research A296, 33 (1990).

\section{DISCLAIMER}

an whensored by an agency of the United States This report was prepared as an account of work sponsored by an agency or thereof, nor any of thei: Government. Neither the United States Government nor any agency thereof, hor any of thability or respoasiemployees, makes any warranty, express or implied, or assumes any legal bility for the accuracy, completeness, or usefulness of any information, apparatus, product, process disclosed, or represents that its use would not infringe privately owned rights. Reference herein to any specific commercial product, precess, or service by trade name, trademark, manufacturer, or otherwise does not necessarily constitute or imply its entorsement, recoms mendation, or frvoring by the United States Government or any agency the and opinions of authors expressed herein do not 

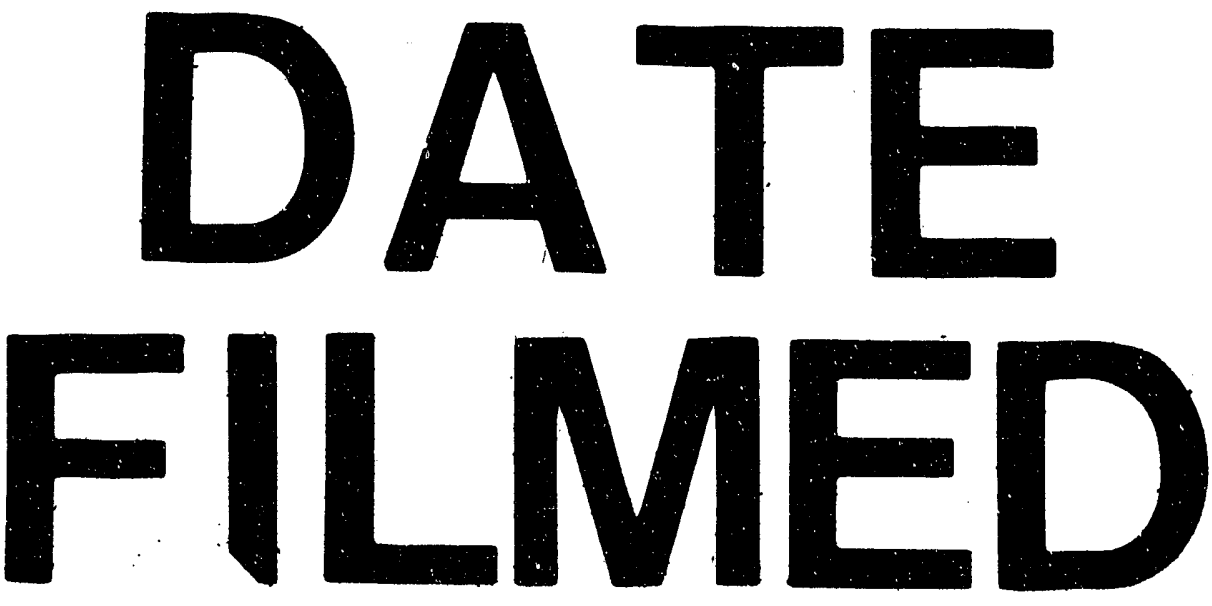

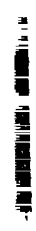

$01 / 20 / 93$ 
